purpose (a concept embodied in the name 'orexin', which implied a role in feeding). The additional roles in sleep-wake regulation and metabolism quickly became clear, but it may be time to revise that view again. Orexin neurons in the medial part of the field show the greatest expression of Fos during wakefulness ${ }^{13}$ and are innervated by brain regions involved with sleep and feeding such as the ventrolateral preoptic area and the ventromedial nucleus of the hypothalamus ${ }^{14}$. In contrast, orexin neurons in the lateral part of the field are activated during conditioned place preference and are more heavily innervated by areas implicated in reward and motivation such as the ventral tegmental area, accumbens nucleus and central nucleus of the amyg- dala $^{14}$. Thus, the medial wing of the orexin population may be driven by metabolic and homeostatic inputs, whereas the lateral wing may receive information related to reward and motivated behaviors. It will be important to determine whether there are differences in the outputs of these fields that reflect these connections and apparent roles. However, by their projections to the cerebral cortex, motor neurons and the extrapyramidal system, both wings of the orexin population may provide an impetus through which motivated states influence whether specific behaviors will be suppressed or expressed.

1. Guilleminault, C., Carskadon, M. \& Dement, W.C. Arch. Neurol. 30, 90-93 (1974).

2. Harris, G.C., Wimmer, M. \& Jones, G.A. Nature, advance online publication 14 August 2005 (doi: 10.1038/nature04071)

3. Crocker, A. et al. Neurology (in the press).

4. Sakurai, T. et al. Cell 92, 573-585 (1998).

5. Winsky-Sommerer, R. et al. J. Neurosci. 24, 11439 11448 (2004).

6. Shalev, U., Yap, J. \& Shaham, Y. J. Neurosci. 21 , RC129 (2001).

7. Piazza, P.V. \& Le Moal, M.L. Annu. Rev. Pharmacol Toxicol. 36, 359-378 (1996).

8. Yamanaka, A. et al. Neuron 38, 701-713 (2003).

9. Mileykovskiy, B.Y., Kiyashchenko, L.I. \& Siegel, J.M. Neuron 46, 787-798 (2005).

10. Lee, M.G., Hassani, O.K. \& Jones, B.E. J. Neurosci. 25, 6716-6720 (2005).

11. Driver-Dunckley, E., Samanta, J. \& Stacy, M. Neurology 61, 422-423 (2003).

12. Hara, J. et al. Neuron 30, 345-354 (2001).

13. Estabrooke, I.V. et al. J. Neurosci. 21, 1656-1662 (2001).

14. Yoshida, K., McCormack, S., España, R., Crocker, A. \& Scammell, T. J. Comp. Neurol. (in the press).

\title{
Object-specific unconscious processing
}

Seeing is not always perceiving. In the visual system, a ventral pathway for conscious perception and a dorsal pathway for unconscious perception are dissociable. However, the evidence for such a dissociation comes mostly from non-human primate studies and rare human patient studies. Now a paper on page 1381 of this issue reports that activation of the unconscious dorsal pathway is object-specific in normal subjects, even when interocular suppression (also called binocular rivalry) had made them completely unaware of the objects being presented. These results are consistent with the idea that areas in the dorsal pathway show object sensitivity, and also indicate that a considerable amount of information from the suppressed eye is processed during binocular rivalry.

Both the ventral and dorsal visual pathways contain object-sensitive areas. The picture shows such areas on a representative brain that has been 'inflated' so that sulcal anatomy is visible on the brain surface: the intraparietal sulcus and V3A/V37 are part of the dorsal pathway, and the lateral occipital cortex (LOC) and temporal areas are part of the ventral pathway. The dorsal object-sensitive areas are known to respond more to objects that can be manipulated (such as tools). How does awareness modulate this response?

Fang and He used an inter-ocular suppression protocol to answer this question. Subjects were scanned while they detected size changes in a central fixation cross. During this detection task, they also saw green-colored objects with low contrast and luminance in one eye, while simultaneously seeing redcolored visual 'texture' (a randomly pixelated image) with high contrast and luminance in the other. The object images were thus entirely suppressed, and not consciously perceived by the subjects, who instead saw only the red visual texture.

However, even when the subjects did not consciously see the suppressed images, dorsal visual stream areas were more active when subjects saw images of tools, compared to when they saw images of faces. In contrast, activity in the ventral visual areas was near baseline during suppression, when subjects could not perceive the objects.

The dorsal pathway therefore responds in a selective manner: it is not activated equally by all unconsciously perceived stimuli, but instead responds more to graspable objects such as tools. This preference may be linked to the important role of the dorsal cortex in controlling reaching and grasping. These results also show that images blocked due to binocular rivalry (generally thought to occur in V1) still have access to later processing, though it is still not clear how such information reaches later stages of the dorsal pathway. In the meantime, these results provide a better understanding of how some patients can use object information without perceiving the objects.

\section{Charvy Narain}

